EPJ Web of Conferences 17, 09004 (2011)

DOI: $10.1051 /$ epjconf/20111709004

(C) Owned by the authors, published by EDP Sciences, 2011

\title{
Evaporation-residue cross sections: role of the entrance channel
}

\author{
Neil Rowley ${ }^{1, a}$ and Nabila Saffidine Grar $^{2}$ \\ 1 UMR 8608, Institut de Physique Nucléaire, 91406 Orsay Cedex, France \\ 2 Département des Sciences de la Matière, Institut de Sciences et Technologie, Centre Universitaire de Bordj Bou Arreridj, \\ 340000 Algeria
}

\begin{abstract}
In the context of the creation of heavy evaporation residues, the interplay between capture, quasifission and true compound-nucleus fission is discussed. An approximate factorisation of the cross section into a compound nucleus term and an entrance-channel term is derived, with each of these terms being clearly defined.
\end{abstract}

\section{Introduction}

The creation of high- $Z$ evaporation residues (ER) via heavyion capture is a complex process. At the first stage of the reaction, the colliding partners must overcome or penetrate an external Coulomb barrier (more generally a distribution of barriers $[1,2])$ for the reaction to proceed. The composite system must then fuse to form an equilibrated compound nucleus $(\mathrm{CN})$. Of course during this second phase, there may be competition from pre-equilibrium re-separation of the two fragments; a system-dependent process referred to as quasifission $(\mathrm{QF})$. Finally, the compound nucleus itself can decay by true fission, rather than becoming a longerlived residue, through the evaporation of neutrons, protons or $\alpha$-particles. The final ER cross section corresponding to this sequence of events - capture, fusion, survival against fission - is sometimes schematically expressed by the equation (see, for example Refs. [3,4]):

$$
\sigma_{\mathrm{ER}}=\sigma_{\text {cap }} P_{\text {fus }} W_{\text {sur }} .
$$

One of the aims of this paper is to suggest a more physically correct version of this formula for the heavy systems for which QF becomes important.

An average, experimental fusion probability has been defined by Sahm et al. [5]:

$$
<P_{\text {fus }}>\equiv \frac{\tilde{\sigma}_{\mathrm{ER}}(E)}{\tilde{\sigma}_{\max }\left(E^{*}\right)},
$$

where $\tilde{\sigma}_{\max }\left(E^{*}\right)$ is the maximum possible reduced cross section for production of evaporation residues from the compound nucleus at an excitation energy $E^{*}$ :

$$
\tilde{\sigma}_{\max }\left(E^{*}\right)=\sum_{L}(2 L+1) W_{\text {sur }}\left(E^{*}, L\right)
$$

The term 'reduced' simply means that the purely kinematic factor $\pi / k^{2} \equiv \hbar^{2} \pi /(2 M E)$ has been omitted from the cross section to give a quantity which is both dimensionless and

\footnotetext{
a e-mail: rowley@ipno.in2p3.fr
}

independent of the reaction through which the compound nucleus is created (with a reduced mass $M$ determined by the target and projectile combination, and at a centre-ofmass energy $E$ ). Note that $\tilde{\sigma}_{\max }$ depends only on the compound nucleus excitation energy $E^{*}$, whereas other quantities will depend on the target and projectile properties. To reflect this, they are written as functions of the appropriate centre-of-mass energy $E$; though of course these two energies are related in a system-dependent way by $E^{*}=E+Q$, where $Q=\left(M_{1}+M_{2}-M_{\mathrm{CN}}\right) c^{2}$ is the reaction $Q$ value.

Although $\tilde{\sigma}_{\max }\left(E^{*}\right)$ is perfectly well defined theoretically, and can in principle be obtained exploiting statisticalmodel (SM) codes, there are many uncertainties involved in such calculations; small variations of the parameters (especially the fission barrier $B_{\text {fiss }}$ ) can lead to large changes in the calculated values of $\tilde{\sigma}_{\max }$. However, as we shall see below, in some cases the reduced cross section might be reliably obtained by comparison of experimental data on different systems, when these exist.

The expression (2) is an excellent way of representing experimental data, and has frequently been employed in the literature [6-9]. These earlier papers used $\tilde{\sigma}_{\max }$ values obtained from SM calculations such as HIVAP [10] and found a roughly constant value of $<P_{\text {fus }}>$ at high energies. This was then normalised to unity, and the decrease of the function at lower energies was attributed to the various entrance-channel effects in question, depending sensitively on the system being studied. There are two reasons for requiring a normalisation: (i) uncertainties in the fission barrier etc., as discussed above and (ii) the effect of quasifission that will be discussed below in Sect. 2 .

Further to the problems of obtaining $\tilde{\sigma}_{\max }\left(E^{*}\right)$, there is the question of how the fusion probability $<P_{\text {fus }}>$ should be interpreted in terms of the reaction dynamics. In Ref. [11] the constant value of this quantity at high energy (well above the Coulomb barriers) was used to quantify an average amount of QF in the system. The object of the present work is to show that it is meaningful to write:

$$
\sigma_{\mathrm{ER}}(E)=<P_{\text {fus }}(E)>\sigma_{\max }\left(E^{*}\right)
$$



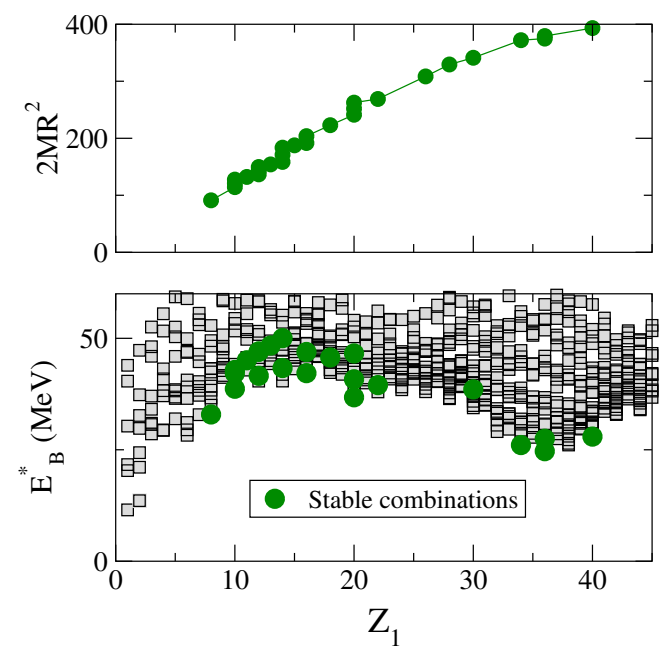

Fig. 1. Filled circles show (top) the inertia parameter $2 M R^{2}$ $\left(\mathrm{amu} \mathrm{fm}{ }^{2} \hbar^{-2}\right.$ ) and (bottom) the excitation energy $E_{B}^{*}$ at the Bass barrier [12] for all possible stable + stable reactions leading to ${ }^{220} \mathrm{Th}$. The grey squares show $E_{B}^{*}$ for many exotic combinations. The projectile charge is $Z_{1}$ and that of the target $Z_{2}=90-Z_{1}$.

Table 1. Some systems leading to ${ }^{220} \mathrm{Th}$. We give the quantities shown in Fig. 1 for a few systems in which good data are available. In addition we quote the Bass barrier $B$ [12], that is seen to vary significantly more than $E_{B}^{*}$. Also shown are the values of $P_{\text {fus }}^{-1}$, where $P_{\text {fus }}$ normalises the high-energy reduced cross section to that for ${ }^{16} \mathrm{O}+{ }^{204} \mathrm{~Pb}$, where QF is believed to be absent.

$\begin{array}{lrcccr}\text { System } & & B(\mathrm{MeV}) & E_{\mathrm{B}}^{*}(\mathrm{MeV}) & 2 M R^{2} & P_{\text {fus }}^{-1} \\ { }^{96} \mathrm{Zr}+{ }^{124} \mathrm{Sn} & {[5]} & 216.3 & 27.97 & 393.4 & 10 \\ { }^{82} \mathrm{Se}+{ }^{138} \mathrm{Ba} & {[9]} & 206.6 & 26.07 & 372.0 & 10 \\ { }^{70} \mathrm{Zn}+{ }^{150} \mathrm{Nd} & {[13]} & 196.5 & 38.57 & 341.2 & 24 \\ { }^{16} \mathrm{O}+{ }^{204} \mathrm{~Pb} & {[3]} & 77.44 & 32.93 & 91.25 & 1\end{array}$

and to show how to interpret $<P_{\text {fus }}>$ theoretically as a function of the incident energy $E$. (Note that the above equation is valid for cross sections or for reduced cross sections, though $\sigma_{\max }$ contains the factor $\pi / k^{2}$ and is, therefore system dependent.)

\section{High-energy limit $\left[T_{L}=1\right]$}

Certain compound nuclei can be produced via many entrance-channel combinations, and in the present work we shall concentrate on various reactions leading to ${ }^{220} \mathrm{Th}$. Fig. 1 shows $2 M R^{2}$ and $E_{\mathrm{B}}^{*}$, the excitation energy of the ${ }^{220} \mathrm{Th}$ compound nucleus formed at the Coulomb barrier (radius $R$ ) with various target-projectile combinations. The target charge is denoted by $Z_{1}$ (thus $Z_{2}=90-Z_{1}$ ) and $E_{\mathrm{B}}^{*}$ is shown for those combinations for which the appropriate mass excesses are known. Stable-stable combinations are shown as filled circles and unstable ones by shaded squares. The particular combinations discussed in this paper are given in Table 1, where we also show the Bass barrier $B$ [12] used in the calculation of $E_{\mathrm{B}}^{*}$. It can be seen that

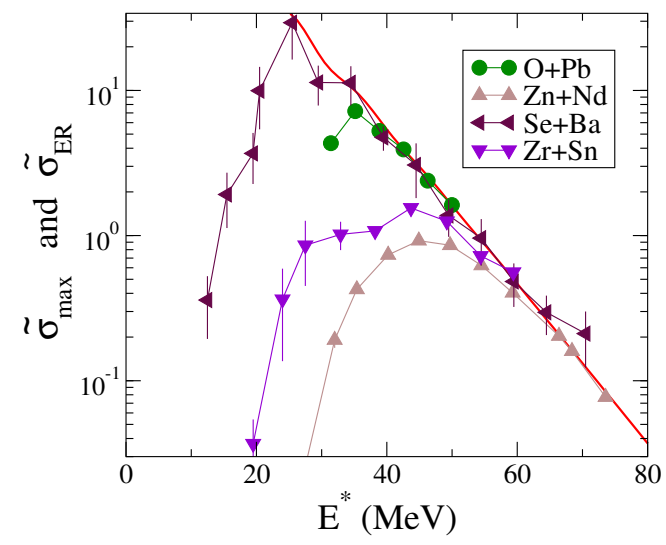

Fig. 2. Experimental reduced evaporation-residue cross section (sum of $x$-n channels) for the reactions shown (mass numbers given in Table 1), normalised to the statistical-model $\tilde{\sigma}_{\max }$ (solid curve) at high excitation energy $E^{*}$. The correct overall normalisation is provided by the ${ }^{16} \mathrm{O}+{ }^{204} \mathrm{~Pb}$ system, which is expected to have little or no quasifission. The ${ }^{82} \mathrm{Se}+{ }^{138} \mathrm{Ba}$ reaction fixes the $E^{*}$ dependence of $\tilde{\sigma}_{\max }$ over a large energy range spanning the barrier regions for ${ }^{96} \mathrm{Zr}+{ }^{124} \mathrm{Sn}$ and ${ }^{70} \mathrm{Zn}+{ }^{150} \mathrm{Nd}$.

the barrier height has a much wider range of values than the excitation energy obtained.

At energies above the highest barrier, the lower angular momenta that are capable of surviving fission will be fully captured. In that case, it is a consequence of the Bohr hypothesis [14] that the reduced cross section for evaporation-residue formation (at a given excitation energy) will be independent of the reaction partners. Berriman et al. show that at such energies (where the barriertransmission coefficients are given by $T_{L}=1$ for all partial waves contributing to the evaporation-residue cross section), the experimental reduced cross sections for this system are all proportional to one another [11] This is interpreted in terms of them all being given by

$$
\tilde{\sigma}_{\mathrm{ER}}(E) \rightarrow P_{\text {fus }} \tilde{\sigma}_{\max }\left(E^{*}\right) .
$$

Since it is believed that there is no QF for the most asymmetric reaction ${ }^{16} \mathrm{O}+{ }^{204} \mathrm{~Pb}\left(P_{\text {fus }}=1\right)$, one can then obtain the fusion probabilities for the other systems. The normalised, experimental, reduced cross sections ${ }^{1}$ are shown in Fig. 2 and the corresponding values of $P_{\text {fus }}^{-1}$ are indicated in Table 1.

Other systems leading to the same $\mathrm{CN}$ have been measured but we restrict our choice here to those in Table 1 in order to demonstrate how important information can be extracted from such comparisons. In Fig. 2, the solid line represents SM results that will be described elsewhere [15]. It essentially provides a curve to which the various sets of experimental data can be normalised. The most asymmetric stable-stable combination possible for this $\mathrm{CN}$ is ${ }^{16} \mathrm{O}+$

\footnotetext{
${ }^{1}$ Rather than looking at the full evaporation-residue cross sections, we shall look at the sum of the $x$-n channels, since the dynamics of the reactions can be distorted by charged-particle emission which can lead to much larger total cross sections through the creation of residues with significantly lower fissilities (lower $\left.Z_{\mathrm{ER}}\right)[5,11]$.
} 
${ }^{204} \mathrm{~Pb}$, for which no normalisation is necessary since there is no QF in that case. However, it provides the behaviour of $\tilde{\sigma}_{\max }$ over a rather limited range of $E^{*}$ since it was not measured to high energy and at the low-energy end it is cut off by the relatively high $E_{\mathrm{B}}^{*}$ (see Table 1 ). The data for ${ }^{82} \mathrm{Se}+{ }^{138} \mathrm{Ba}$ have larger statistical errors, but extend over a very large energy range $\left(12<E^{*}<70 \mathrm{MeV}\right)$ providing a well defined $\tilde{\sigma}_{\max }$ that can be used to discuss the other two systems of Table 1 . These both have good quality data that show deviations from the maximum cross section over a large range of energies, highlighting the role of the entrance channel.

\section{Large reduced mass and no quasifission}

Ignoring quasifission we may write:

$$
\tilde{\sigma}_{\mathrm{ER}}(E)=\sum_{L}(2 L+1) T_{L}(E) W_{\mathrm{sur}}\left(E^{*}, L\right),
$$

and since the transmission coefficients $T_{L}(E)$ depend only on the energy relative to the Coulomb barrier, we may also write

$$
T_{L}(E) \approx T_{0}\left(E-\frac{L(L+1)}{2 M R^{2}}\right) .
$$

Expanding this expression to first order in $L(L+1)$ allows us to derive [15]

$$
\tilde{\sigma}_{\mathrm{ER}}(E) \approx T_{0}(E-\Delta E) \tilde{\sigma}_{\max }\left(E^{*}\right),
$$

where the energy shift is given by

$$
\Delta E \approx \frac{<L(L+1)>}{2 m R^{2}} .
$$

The values of $<L(L+1)>$ depend only on $E^{*}$ and are averages over the relevant $\mathrm{CN}$ survival probabilities. They can be simply obtained from HIVAP calculations and are much less sensitive to the parameters than the maximum cross section. In the vicinity of the barriers for all of the reactions considered here, we find $\langle L(L+1)>\approx 300$. The maximum shift will then clearly be found in the system with the lowest reduced mass, that is, ${ }^{16} \mathrm{O}+{ }^{204} \mathrm{~Pb}$ for which Eq. (8) gives $\Delta E \approx 2.9 \mathrm{MeV}$.

\subsection{Experimental shift in the absence of QF}

For this system, good data exist both on the capture cross section and on the fusion- $x \mathrm{n}$ reaction [3]. With good enough data one can obtain approximate values of the $s$-wave transmission coefficient from $[2,16]$

$$
T_{0} \approx \frac{1}{\pi R^{2}} \frac{d\left(E \sigma_{\mathrm{cap}}\right)}{d E}
$$

without using coupled-channels calculations. In Fig. 3 we compare the experimental values of $T_{0}$ (from the above equation) with $\sigma_{\mathrm{ER}} / \sigma_{\max }$ and see that the asymmetric system ${ }^{16} \mathrm{O}+{ }^{204} \mathrm{~Pb}$ does indeed show the relative shift predicted by Eqs. $(7,8)$. We note that for the more symmetric systems given in Table 1 , any shifts will be significantly smaller than this value $(<1 \mathrm{MeV})$. They will be ignored in the rest of this paper.

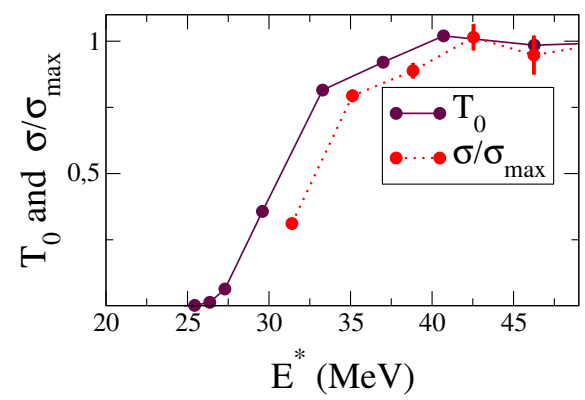

Fig. 3. Experimental data for the system ${ }^{16} \mathrm{O}+{ }^{204} \mathrm{~Pb}$ show the predicted shift (Eqs. $(7,8))$ between the $s$-wave transmission coefficient (from capture) and the ratio $\sigma_{\mathrm{ER}} / \sigma_{\max }$.

\section{Putting back the QF probabilities}

For a constant value of $P_{\text {fus }}$ we obtain

$$
\tilde{\sigma}_{\mathrm{ER}} \approx T_{0}(E) P_{\text {fus }} \tilde{\sigma}_{\max }\left(E^{*}\right) .
$$

This generalises the results of Berriman et al. to the energy region where $T_{L}<1$, but the constancy of the fusion probability should be relaxed for systems where QF is important. In terms of the capture barrier distribution we have [2]

$$
T_{0}(E)=\sum_{\alpha} w_{\alpha} T_{0}\left(E-B_{\alpha}\right) \equiv \sum_{\alpha} w_{\alpha} T_{0}^{\alpha}(E),
$$

where $B_{\alpha}$ and $w_{\alpha}$ are the heights and weights of the entrance-channel barrier distribution. In the spirit of the very successful di-nuclear system (DNS) model $[17,18]$, we may consider the QF to be dependent upon the entrance-channel configuration, whereupon Eq. (10) generalises to

$$
\tilde{\sigma}_{\mathrm{ER}} \approx<P_{\text {fus }}(E)>\tilde{\sigma}_{\max }\left(E^{*}\right)
$$

with the average fusion probability given by

$$
<P_{\text {fus }}(E)>=<T P>\equiv \sum_{\alpha} w_{\alpha} T_{0}^{\alpha}(E) P_{\text {fus }}^{\alpha} .
$$

This achieves the desired factorisation of the evaporationresidue cross section into $\mathrm{CN}$ and entrance-channel terms.

\section{Fusion barrier distribution}

The barrier distribution for capture is given by $[1,2]$

$$
D_{\text {capture }}=\frac{1}{\pi R^{2}} \frac{d^{2}\left(E \sigma_{\text {capture }}\right)}{d E^{2}} \equiv \frac{d T_{0}}{d E},
$$

with $T_{0}$ defined by Eq. (9). This has often been referred to as the experimental "fusion" barrier distribution. However, if we wish to obtain a barrier distribution relating to the formation of a heavy compound nucleus, then we must include the possibility of QF and define a "true" fusion barrier distribution by

$$
D_{\text {fus }}=\frac{d<P_{\text {fus }}>}{d E} .
$$




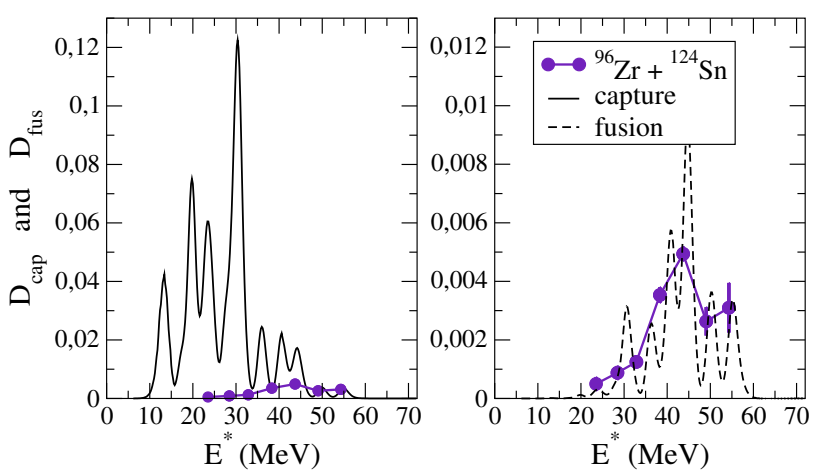

Fig. 4. Left-hand panel: capture and fusion barrier distributions for the ${ }^{96} \mathrm{Zr}+{ }^{124} \mathrm{Sn}$ system. The fusion curve is obtained from the data using Eq. (15). The capture distribution is calculated using CCFULL [19] with two octupole phonons in ${ }^{96} \mathrm{Zr}$, and one quadrupole phonon and two octupole phonons in ${ }^{124} \mathrm{Sn}$. The calculations use the known excitation energies and deformation parameters from Refs. [20,21]. Right-hand panel: the theoretical capture distribution is mapped onto the fusion data with the barrier-dependent fusion probability discussed in the text.

Fig. 4 shows the experimental values of $D_{\text {fus }}$ from the ${ }^{96} \mathrm{Zr}$ $+{ }^{124} \mathrm{Sn} x$-n data and compares them with the theoretical $D_{\text {capture }}$ (see figure caption for details of the coupledchannels calculations). Of course the normalisations of the curves differ by the Berriman factor of 10 (see Table 1) due to quasifission.

The most important observation on Fig. 4 is that $D_{\text {fus }}$ falls off at the top of the capture barrier distribution. This is also true for the ${ }^{70} \mathrm{Zn}+{ }^{150} \mathrm{Nd}$ system (not shown), and indeed for all other systems studied [15], and in all cases $D_{\text {fus }} \leq D_{\text {capture }}$ at all energies. This implies that fusion is indeed determined by the capture barriers, with a transmission modified by barrier-dependent quasifission. We can now deduce this barrier dependence, since it transforms the solid line in the left-hand panel of Fig. 4 to the experimental data. The precise form of the transformation is unimportant but the basic physical properties of the $\mathrm{QF}$ are readily summarised by multiplying $D_{\text {capture }}$ by a Fermi function $\left.P_{\text {fus }}^{\alpha} \equiv P_{\text {fus }}(B)=P /\left[1+\exp \left(\left(B-B_{0}\right) / \Delta B\right)\right)\right]$. The right-hand panel shows that one can reasonably reproduce the data with $P=1.0, B_{0}=45 \mathrm{MeV}$ and $\Delta B=4 \mathrm{MeV}$. This means that for the very highest barriers in this system (around $50<E^{*}<55 \mathrm{MeV}$ ) there is almost $100 \%$ fusion, but this falls off rapidly for the lower-energy barriers.

For ${ }^{70} \mathrm{Zn}+{ }^{150} \mathrm{Nd}$ the equivalent transformation has $P=0.2, B_{0}=52 \mathrm{MeV}$ and $\Delta B=9 \mathrm{MeV}$; thus there is only $20 \%$ fusion even for the highest barriers and a slower fall off for lower $B$. The different behaviour in these and other systems will be discussed in detail elsewhere [15].

\section{Conclusions}

The creation of evaporation residues is a time-ordered sequence of events in which the colliding ions are first captured, then evolve towards an equilibrated compound nucleus, which finally may or may not survive against true fission. We have shown that for heavy systems, a good factorisation of the expression for the ER cross section is

$$
\sigma_{\mathrm{ER}} \approx<P_{\text {fus }}>\sigma_{\text {max }},
$$

where the quantities on the right-hand side are both well defined. Note that the compound-nucleus dependence factors out completely from an entrance-channel term. However, the latter does not factorise into separate capture and fusion terms. Indeed it is the barrier-dependent fusion probability averaged over the $s$-wave transmissions for each barrier. This leads naturally to a constant value above the highest barrier, as found in Ref. [11]. The above equation is somewhat different from the expression (1), which is at best schematic, and in which the survival probability and the fusion probability are ill-defined averages.

The more correct form of this equation allows the simple extraction of the barrier dependence of the quasifission from sufficiently good experimental data. Possible mechanisms for this dependence will be discussed elsewhere [15].

\section{References}

1. N. Rowley, G.R. Satchler and P.H. Stelson, Phys. Lett. B254, (1991) 25

2. M. Dasgupta, D.J. Hinde, N. Rowley, and A.M. Stefanini, Ann. Rev. Nucl. Part. Sci. 48, (1998) 401

3. D.J. Hinde, M. Dasgupta and A. Mukherjee, Phys. Rev. Lett. 89, (2002) 282701

4. R.S. Naik et al., Phys. Rev. C 76, (2007) 054604

5. C.C. Sahm et al., Nucl. Phys. A441, (1985) 316

6. A.B. Quint et al., Z, Phys. A346, (1993) 119

7. J.G. Keller et al., Nucl. Phys. A452, (1986) 173

8. K.-H. Schmidt and W. Morawek, Rep. Prog. Phys. 54, (1991) 949

9. K. Satou et al., Phys. Rev. C 65, (2002) 054602

10. W. Reisdorf, Z. Phys. A300, (1981) 227; W. Reisdorf and M. Schädel, Z. Phys. A343, (1992) 47

11. A.C. Berriman et al., Nature Vol. 413, (2001) 144

12. R. Bass, Phys. Rev. Lett. 39, (1977) 265

13. C. Stodel, PhD thesis, LPC Caen (1998)

14. N. Bohr, Nature 137, (1936) 344

15. N. Saffidine Grar and N. Rowley, in preparation

16. A.B. Balantekin and N. Takigawa, Rev. Mod. Phys. 70, (1998) 77; L.F.Canto, P.R.S.Gomes, R.Donangelo, M.S.Hussein, Phys. Rep. 424, (2006) 1

17. V.V. Volkov, Izv. AN SSSR, Ser. Fiz. 50, (1979) 1879

18. N.V. Antonenko et al., Phys. Rev. C 51, (1995) 2635; G.G. Adamian, N.V. Antonenko, W. Scheid and V.V. Volkov, Nucl. Phys. A627, (1997) 361; ibid A633, (1998) 409; G.G. Adamian, N.V. Antonenko and W. Scheid, Nucl. Phys. A678, (2000) 24; Phys. Rev. C 69, (2003) 034601

19. K. Hagino, N. Rowley and A.T. Kruppa, Comp. Phys. Comm. 123, (1999) 143; for updates see http://www.nucl.phys.tohoku.ac.jp/hagino/ccfull.html

20. S. Raman et al., Atomic Data and Nuclear Data Tables 36, (1987) 1

21. T. Kibédi and R.H. Spear, Atomic Data and Nuclear Data Tables 80, (2002) 35 